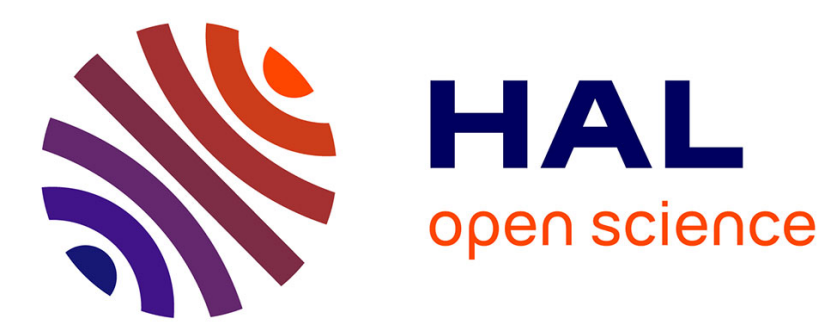

\title{
Fabrication de pâtes à papiers à base de bois feuillus
} Jean Venet

\section{To cite this version:}

Jean Venet. Fabrication de pâtes à papiers à base de bois feuillus. Revue forestière française, 1954, 5, pp.297-310. 10.4267/2042/26992 . hal-03384238

\section{HAL Id: hal-03384238 \\ https://hal.science/hal-03384238}

Submitted on 18 Oct 2021

HAL is a multi-disciplinary open access archive for the deposit and dissemination of scientific research documents, whether they are published or not. The documents may come from teaching and research institutions in France or abroad, or from public or private research centers.
L'archive ouverte pluridisciplinaire HAL, est destinée au dépôt et à la diffusion de documents scientifiques de niveau recherche, publiés ou non, émanant des établissements d'enseignement et de recherche français ou étrangers, des laboratoires publics ou privés. 


\section{REVUE FORESTIERE FRANÇAISE}

1954

MAI

$\mathrm{N}^{\circ} 5$

Dans ce numéro: J. VenET: Fabrication de pâtes à papiers à base de bois feuillus. - J. Campredon et J. Villik̀re: Etudes sur le bois de Peuplier Robusta. - Ph. Duchaufour: Note sur l'influence de l'incinération sur l'évolution de l'humus. - J. Guinaudeau: Germination du Pin Maritime (Résultats des expériences réalisées par l'annexe de la Station de Recherches de Bordeaux).

\section{FABRICATION DE PATES A PAPIERS A BASE DE BOIS FEUILLUS}

Dans le cadre des enquêtes en vue de l'accroissement de la productivité, une mission française s'est rendue aux Etats-Unis en avrilma I952 et son rapport a été publié par l'Union Syndicale des Fabricants de papiers, I64, boulevard Haussmann, Paris ( 8 e).

On sait que l'industrie française des papiers et cartons est suffisamment outillée pour faire face aux besoins actuels de notre pays, mais qu'elle est tributaire de l'étranger pour une part importante de ses approvisionnements. La production française de bois résineux, en particulier Sapin et Epicéa, est insuffisante, ce qui a de graves conséquences: pénurie de papier en période de guerre et constantes hémorragies de devises. L’U.R.S.S. a momentanément cessé ses exportations de bois de papeterie et les autres pays, riches en bois de papèterie résineux, préfèrent exporter des pâtes et des papiers plutôt que des bois. Ceci aggrave d'autant les charges qui pèsent sur notre balance commerciale.

D'autre part, la forêt française est riche en bois feuillus, provenant de taillis ou de jeunes éclaircies de futaie, qu'elle a de plus en plus de mal à écouler comme bois de feu ou comme bois de mine.

Le problème du remplacement des bois résineux par des feuillus pour la fabrication des pâtes à papier ou de cellulose est donc un problème urgent à résoudre et important économiquement. Ces études sont d'ailleurs menées de front avec celles relatives à une utilisation accrue de végétaux annuels, en particulier de paille.

Le même problème se pose aux Etats-Unis, c'est pourquoi une mission, comprenant des fonctionnaires et industriels français, s'est rendue dans ce pays en avril-mai I952 dans le but d'étudier les points suivants :

- savoir si les bois feuillus utilisés en papeterie aux Etats-Unis sont semblables ou non aux bois feuillus français, en vue de se fáre une opinion sur les possibilités de substitution de ces derniers aux bois résinewx, 
- améliorer les connaissances relatives aux procédés classiques de fabrications de pâtes à papier à partir de bois feuillus,

- étudier les méthodes nouvelles de traitement des bois feuillus,

- voir comment ces pâtes de bois feuillus peuvent être incorporées dans les différentes sortes de papier,

- examiner la rentabilité de ces diverses opérations.

\section{Situation ACTUELLE DE L'INDUSTRIE PAPETIÈRE FRANÇAISE}

Quelle est exactement la situation de la France en ce qui concerne la consommation de papiers et cartons, pâtes à papier et bois de papeterie ?

En I95I, la France a produit I 560000 tonnes de papiers et cartons, ce qui représente près de $40 \mathrm{~kg}$ par habitant, à savoir $7 \mathrm{~kg}$ de papier journal, I I $\mathrm{kg}$ d'impression écriture, II $\mathrm{kg}$ d'emballage, $8,5 \mathrm{~kg}$ de carton et $2 \mathrm{~kg}$ de minces et spéciaux. La production a été à peu près égale à la consommation; importations et exportations de papiers et cartons se compensant.

Ces papiers et ces cartons ont été faits en grande partie avec des pâtes fabriquées en France, mais aussi avec des pậtes importées. Par ailleurs, une certaine proportion des pâtes préparées dans notre pays sont faites avec des bois étrangers.

La figure suivante indique, par catégories de pâtes, le pourcentage de notre production par rapport à notre consommation et le pourcentage de bois français par rapport aux bois totaux utilisés pour chaque fabrication.

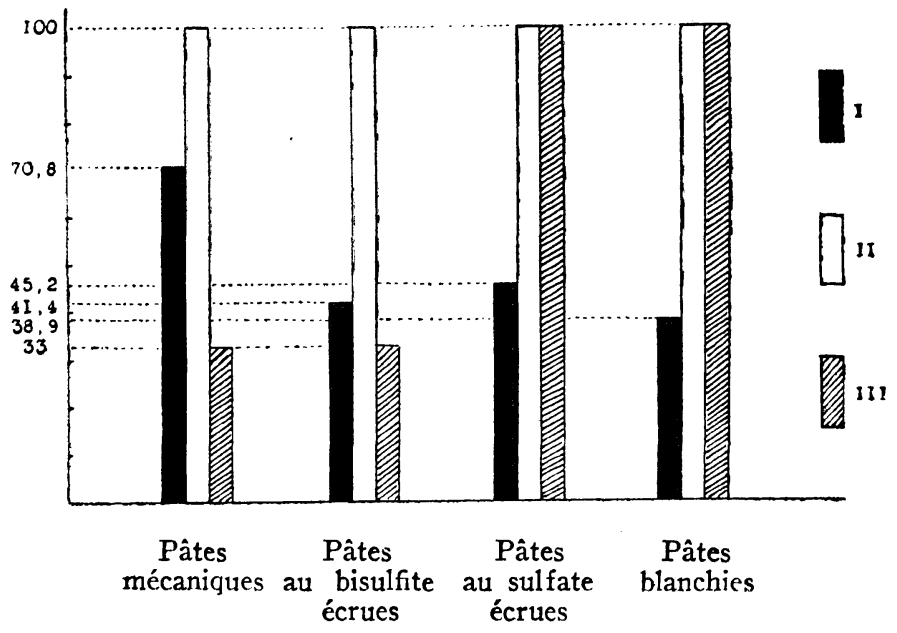

I. Production. - II. Consommation. - III. Pourcentage de bois français par rapport au total: bois français + bois importés. 
La ressource annuelle actuelle de bois de papeterie français est évaluée comme suit par le rapport susvisé (évaluation qui nous semble optimiste):

600 à 800000 stères d'Epicéa et Sapin;

700 à 800000 stères de Pin maritime (plus délignures);

200 à 400000 stères de Pins et autres résineux,

200 à 300000 stères de Tremble, sous réserve de trier cette essence, dans les coupes où elle est mélangée à d'autres bois, 500000 stères de Peuplier,

360000 stères de Châtaignier (quantité réellement utilisée en I95I),

et enfin vastes ressources d'autres feuillus, surtout en charbonnette (diamètre 3 à $6 \mathrm{~cm}$ ) et petits rondins (diamètre 6 à $\mathrm{Io} \mathrm{cm}$ ), mais en moindre quantité en gros rondins (diamètre ro à $20 \mathrm{~cm}$ ) ou petites grumes (diamètre 20 à $30 \mathrm{~cm}$ ).

Jusqu'à ce jour, les quantités de feuillus réellement utilisées (Châtaignier mis à part) ont été assez faibles.

En I95I, par exemple, on n'a utilisé que 7 I 000 stères de feuillus divers, dont 52000 stères de Tremble, contre 869000 stères de Sapin et Epicéa (français), 70 o00 stères de Pin sylvestre, 930000 stères de Pin maritime (I tonne ayant été comptée comme 2,5 stères), 357000 stères de Châtaignier, et 890000 stères d'Epicéa étranger importé. 7I 000 stères ne représentent évidemment que $2,2 \%$ de la consommation totale de bois à pâtes en France. Mais en $v$ incluant le Châtaignier, on peut dire que I3 \% des bois consommés en I95I par l'industrie française des pâtes furent des feuillus. Ce pourcentage est même de $18 \%$ si on ne tient compte que des bois français.

Situation DE L'industrie Papetière aUx U.S.A.

La situation aux Etats-Unis est la suivante:

$$
\begin{array}{cc}
\text { Production américaine } & \text { Importations } \\
\text { (U.S.A.) } & \text { américaines }
\end{array}
$$

Bois de papeterie..

90000000 stères

Io 000000 stères

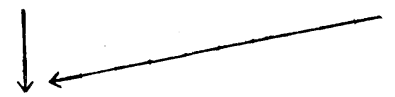

Pâtes à papier .....

I5 000 oo tonnes

I 000 ooo tonnes

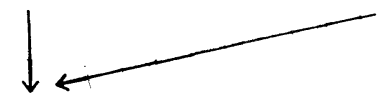

Papiers et cartons ..

Consommation de papiers et cartons ..

27700000 tonnes pour I 50 millions d'habitants, soit $185 \mathrm{~kg}$ par habitant. 
De 1939 à I945, la consommation et la production de papiers et cartons aux U.S.A. a augmenté de façon considérable. La consommation de bois à pâtes, par exemple, est passée de 40 à 100 millions de stères et la consommation de pâtes de 8 à I6 000000 de tonnes. La plus forte augmentation de consommation a porté sur les pâtes au sulfate et sur les produits d'emballage (papiers et cartons).

La production de pâtes à partir de bois feuillus représente aux Etats-Unis $\mathrm{I} 4 \%$ de la production totale de pâtes: ceci correspond à une consommation annuelle de I I millions de stères. Les' usines traitant les bois feuillus sont presque toutes situées au voisinage de la frontière canadienne où elles consomment des bouleaux, trembles et érables. Quelques-unes, implantées plus au Sud, traitent les tulipiers et les gums.

Le tableau suivant donne, pour les principales espèces feuillues, la consommation en I95 I aux Etats-Unis et une évaluation sommaire de la " ressource ) (c'est-à-dire de la fraction du volume total sur pied, capable de donner des bois à fibres).

$$
\begin{array}{cc}
\begin{array}{c}
\text { Consommation } \\
\text { en }
\end{array} & \begin{array}{c}
\text { Ressource } \\
\text { en }
\end{array} \\
\text { millions de stères } & \text { millions de stères }
\end{array}
$$

Bouleaux :

Betula papyrifera

Betula lutea

$\mathrm{r}, 5$

40

Trembles:

Populus tremuloides

Populus grandidentata

200

Erables :

Acer saccharum

Acer rubrum

I

200

Hêtre:

Fagus grandifolia $\quad 0,4 \quad$ non indiquée

Peupliers :

Section tacamahaca

$P$. balsamifera

$P$. candicans

Section aigeiros

ssp angulata

$P$. deltoïdes missouriensis monilifera 
Liquidambar :

L. styraciflua

Tupelo:

Nyssa sylvatica

Tulipier :
1,5

I

100

\section{Liriodendron tulipifera}

0,7

100

Ces feuillus proviennent, en majorité, de la forêt du Nord-Est des Etats-Unis dont certaines parties, les plus belles, contiennent une forte proportion (environ 50 \%) de feuillus: érables, bouleaux, chênes, frênes, hêtres et tillleuls, le reste étant des résineux, tandis que d'autres, qui ont été plus sévèrement exploitées et incendiées, sont presque uniquement boisées d'essences feuillues: trembles, $\cdot$ bouleaux, etc... Les deux tiers des bois de papeterie feuillus proviennent des forêts nordiques.

L'autre source de bois feuillus pour la papeterie est la forêt méridionale, de la Virginie à la Louisiane qui, à côté des pins, contient des feuillus tels que Liquidambar, Tupelo, Tulipier, etc...

Les coupes réalisées dans ces massifs qui, pour la plupart, sont particuliers (avec quelques vastes domaines appartenant à des compagnies papetières) sont, soit des coupes de dimension (enlevant, par exemple, tout ce qui fait plus de Io à $20 \mathrm{~cm}$ de diamètre à hauteur d'homme), soit des coupes à blanc, soit des coupes sélectives (à caractère plus ou moins jardinatoire), soit des éclaircies (assez rarement):

Le rapport entre le prix sur pied du stère de feuillus sous écorce et du stère de résineux varie de 25 à $55 \%$ suivant qu'il s'agit de fournitures riches en trembles ou riches en feuillus durs; il va même jusqu'à 7o \% dans les régions forestières du Sud-Est.

A titre indicatif, les prix du stère sur pied des bois résineux s'échelonnent entre 550 (pins) et $900 \mathrm{~F}$ (sapins et épicéas) et ceux des feuillus entre 200 (tremble) et $500 \mathrm{~F}$ (bouleaux, érables, etc...).

Les frais de main-d'œuvre varient peu dans le Nord, d'une région à l'autre. Le salaire minimum garanti des bûcherons est de $300 \mathrm{~F}$ de l'heure. Les bûcherons, groupés en petites équipes munies de scies mécaniques, font 5 à 7 stères par jour et débardent les bois au moyen d'un cheval prêté par l'exploitant.

Le prix moyen de l'abattage et du débardage varie, par contre, de I 100 - I 650 F par stère, dans le Nord, à 500 - 750 F dans le Sud. Dans le Nord, les coupes sont éloignées des villages et il est 
souvent nécessaire d'y établir des camps. La main-d'œuvre est en outre payée beaucoup moins cher dans le Sud.

L'écorçage à la main est très coûteux: 300 à $450 \mathrm{~F}$ par stère. Il existe des machines portatives pour faire ce travail, mais souvent on ne le fait qu'à l'usine.

L'écorçage chimique semble avoir pris une certaine vogue. Il se réalise de la façon suivante: à la montée de sève, on pratique une annélation circulaire de l'écorce, sur to à $30 \mathrm{~cm}$ à la base de l'arbre et l'on applique immédiatement un poison, tel que l'arsénite de soude, sur le cambium encore humide. Un homme incise, deux font l'annélation et un quatrième badigeonne. Huit mois après, l'écorce se décolle toute seule. Ce type d'écorçage revient deux fois moins cher qu'à la main. Plus l'arbre est gros, moins ce procédé, relativement, coûte cher. Il n'est cependant pas sans danger pour le personnel et le gibier. Ce procédé réussit bien, paraît-il, avec le Hêtre, le Bouleau jaune, le Sapin, mais moins bien avec les Erables, le Bouleau blanc, le Frêne ou le Tsuga. D'autres produits que l'arsénite de soude (hormones, par exemple) sont à l'essai.

Le prix du transport vers l'usine varie de $45^{\circ}$ à I $200 \mathrm{~F}$ par stère, suivant densité des bois et distances. De ce fait, les prix de revient du stère écorcé, rendu usine, s'établissent approximativement comme suit :

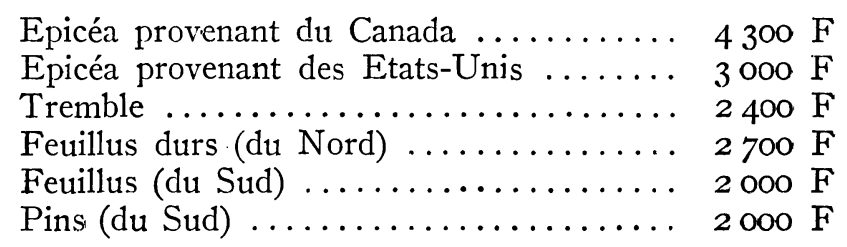

Bien que ces prix de revient soient avantageux (il semble d'ailleurs, par comparaison avec les chiffres qui précèdent, qu'il s'agisse de prix minima), on cherche encore à les restreindre, soit par le transport des tiges sur toute leur longueur (pour débit et écorçage en usine), soit, au contraire, en réduisant le bois en copeaux en forêt même.

Certains bois américains sont assez semblables aux bois homologues français: Pins du Sud, Trembles; d'autres sont un peu supérieurs: Bouleaux et Erables; d'autres, par contre, sont inférieurs : Sapin baumier et Pin de Banks.

Selon les études de laboratoire, les Epicéas, Sapins, Tsugas, Mélèzes et Pins du Nord-Est des Etats-Unis ont des fibres de l'ordre de 3,5 $\mathrm{mm}$ de long contre 5 à $7 \mathrm{~mm}$ pour les Epicéas, Sapins, Douglas, Mélèzes et Sequoias de l'Ouest. Les Pins jaunes du Sud ont 
des fibres de $4,5 \mathrm{~mm}$. La longueur des fibres des feuillus américains va de I à I,25 $\mathrm{mm}$ pour les Châtaigniers, Erables à sucre, Frênes, Tilleuls, Boùleaux à papier, Hêtres, Trembles, à I,50 ou I,80 mm pour les Bouleaux jaunes, Ormes, Liquidambars et Tulipiers.

Selon l'Institut National du Bois, les longueurs des fibres des feuillus français vont de 0,7 à $1,05 \mathrm{~mm}$ pour les Aunes, Frênes, Châtaignier, Chênes, Ormes et Tremble, à I, Io ou même I,60 mm pour les Hêtre, Tilleuls et Charme. Ce dernier, avec r, $6 \mathrm{~mm}$, se classe au même rang que les bois feuillus américains, comme le Liquidambar.

A noter que la grosseur des bois de papeterie est sensiblement plus élevée aux U.S.A. qu'en France: les trembles, par exemple, ont 20 à $25 \mathrm{~cm}$ de diamètre contre Io à I 5 en moyenne chez nous. Ceci est important.

Les procédés de fabrication sont, en général, les mêmes que dans notre pays. On fait de la pâte mécanique avec l'Epicéa, pour le papier journal, et le Tremble, pour le " tissue-paper ) (essuie-mains, papier mousseline), le papier à machine et même l'impression écriture.

Pour les pâtes chimiques an sulfate et à la soude (écrues ou blanchies), on utilise les Pins du Sud et les feuillus.

Tous les feuillus peuvent donner des pâtes au sulfate écrues qui conviennent bien pour le papier mousseline et le papier à imprégner.

Les pâtes au sulfate blanchies, faites avec des feuillus, sont légèrement moins solides que les pâtes d'Epicéa au bisulfite, mais néanmoins fort utilisables en mélange, apportant des qualités de douceur, moelleux, " main ), opacité et " épair ).

Les pâtes à la soude blanchies (en régression par rapport aux pâtes au sulfate) avaient les mêmes avantages.

On fait par le procédé au bisulfite avec les feuillus, soit des pâtes blanchies, soit des pâtes écrues (mais dans ce cas, il s'agit de Tremble mélangé à l'Epicéa dans une proportion de 5 à I $5 \%$ ). Les produits obtenus ont une moindre résistance à l'éclatement ou à la déchirure mais, en mélange avec les pâtes de résineux, ils améliorent la formation et la surface de la feuille. Ils s'emploient aussi pour le papier cristal et la viscose.

Les feuillus conviennent particulièrement bien pour les pâtes mi-chimiques (sulfite neutre sur copeaux, sulfite neutre sur rondins, soude froide sur copeaux) où l'on conjugue une action chimique et une action mécanique.

Le procédé au sulfite neutre sur copeaux est industriel. La pâte ainsi faite s'engraisse bien, mais elle est difficile à égoutter sur ma- 
chine.- Les produits obtenus manquent d'opacité mais ne sont que très faiblement poreux (avantage pour les emballages alimentaires, gobelets, papiers carbone).

Selon les essais de laboratoire, les propriétés comparées des feuillus et résineux peuvent se résumer comme suit:
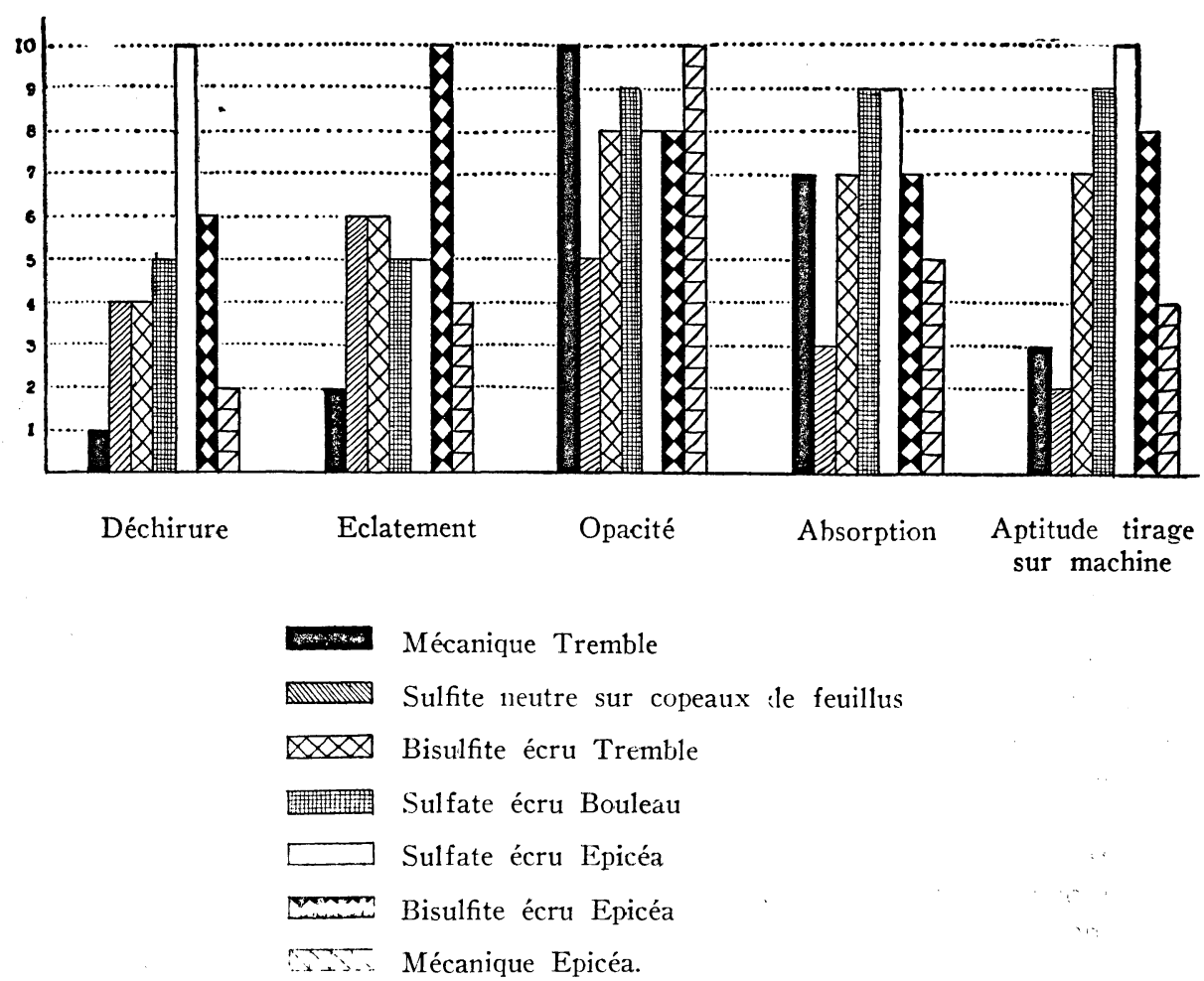

La pâte de feuillus, avec des faiblesses qui ne sont pas des vices rhédibitoires, apporte donc aux papiers et cartons certaines qualités particulières qui rendent son emploi possible, et même souhaitable, en mélange avec les pâtes de bois résineux.

Il y a lieu toutefois de tenir compte du prix de revient des pâtes de bois feuillus. Les prix limites des pâtes produites aux Etats-Unis variaient, en $195^{2}$, de $37000 \mathrm{~F}$ la tonne, pour la pâte mécanique, à $58000 \mathrm{~F}$ pour la pâte au sulfate blanchie. L’Américain est un gros consommateur de papier ( par habitant $44 \mathrm{~kg}$ de papier journal; 
ro $\mathrm{kg}$ de ( tissue-paper », etc...) mais les prix de ces divers papiers ou cartons ne représentent, aux Etats-Unis, que le tiers ou la moitié des prix français.

A grosseur égale, les rondins d'Epicéa, rendus usine, valent 50 à $60 \%$ plus cher en France qu'aux U.S.A., le Tremble $40 \%$, les feuillus durs $25 \%$ (ou le même prix dans le Nord), le Pin maritime I25 à I $50 \%$ (2 à 2,5 fois) plus cher. A noter, au sujet de ce dernier chiffre, que la main-d'œuvre dans le Sud des Etats-Unis est beaucoup moins payée que dans le Nord, et aussi que l'exploitation des coupes y est moins coûteuse qu'en France, parce qu'elle est moins soignée que celle de notre forêt de Pin maritime.

Les rendements en pâte varient de 5,2 à 9 stères pour une tonne de pâte, suivant les essences. Toutes choses égales d'ailleurs (grosseur et siccité, par exemple), ces rendements sont comparables à ceux des bois français.

Les produits chimiques valent en général plus cher qu'en France: soufre $122 \%$ en plus, soude $24 \%$, chlore $56 \%$. La consommation de produits chimiques par tonne de pâte est, en outre, sensiblement plus faible aux U.S.A. qu'en France (surtout pour le sulfate de sodium). L'énergie électrique coûte le tiers et le charbon est deux à trois fois moins cher. Les salaires industriels sont élevés : I,4 à 2,75 dollar par heure (avec seulement Io \% de charges sociales). Mais le nombre d'heures de main-d'œuvre par tonne de pâte est 3 à 4 fois plus faible, non pas pour des raisons humaines, mais pour des raisons de mécanisation.

- La comparaison entre feuillus et résineux montre que le prix de revient des résineux (pâte chimique $=$ Ioo) est plus élevé que celui des feuillus (pâte chimique $=65$, pâte mi-chimique $=50$, pâte mécanique $=50$ ), pour des raisons de rendement au stère de bois et de frais de fabrication.

Ce qui vient d'être dit explique pourquoi les feuillus ont commencé à prendre une place importante dans la fabrication des pâtes à papier aux Etats-Unis. Trembles et Peupliers servent à faire 25 à $30 \%$ des pâtes mécaniques. Liquidambar, Tupelo, Tulipier, etc... donnent 90 à $95 \%$ des pâtes mi-chimiques. Les mêmes essences, avec les Bouleaux, les Erables et le Hêtre, donnent Io à I $5 \%$ des pâtes à la soude et au sulfate blanchies et 2 à $3 \%$ des pâtes écrues: 5 à ro $\%$ des pâtes au bisulfite blanchies viennent des Trembles. Peupliers, Bouleaux, Erables et du Hêtre.

\section{Quelles CONCLUSIONS PEUT-ON EN TIRER POUR LA France ?}

Selon les statistiques établies sous l'égide de l'Organisation des Nations-Unies, les besoins en bois à pâtes de l'Europe (U.R.S.S. non comprise) seront, en I 960 , de l'ordre de 56 à 74 millions de $\mathrm{m}^{3}$, 
suivant les hypothèses faites quant à l'augmentation de la production et à l'évolution du prix des bois (*).

Or, la production de 1'Europe en bois à pâtes, en I960, en admettant que la politique forestière demeure inchangée, sera de l'ordre de 40 millions de $\mathrm{m}^{3}$, auxquels s'ajouteront des déchets de scierie en quantités non chiffrées.

Le déficit en bois à pâtes n'est donc pas seulement français, mais européen et, même si l'on s'oriente résolument vers une organisation européenne de l'économie et des ressources, le problème de l'augmentation de la production des bois à pâtes se pose. Il se pose tout particulièrement pour les pays à forte population, très déficitaires en bois à pâtes et ayant la possibilité d'accroître facilement ou d'améliorer leurs surfaces boisées, ce qui est le cas pour la France.

La même statistique des Nations-Unies reconnaît que les I I millions d'hectares de forêts de la France sont particulièrement bien aménagés. Leur volume sur pied, de l'ordre de 700 millions de $\mathrm{m}^{3}$, produit annuellement quelque 25 à 30 millions de $\mathrm{m}^{3}$, soit $9 \%$ de la production européenne. Mais il s'agit en majeure partie de bois de feu et de grumes à sciages (spécialement d'essences feuillues). La répartition, par catégories, est la suivante, en millions de $\mathrm{m}^{3} \mathrm{de}$ bois rond:

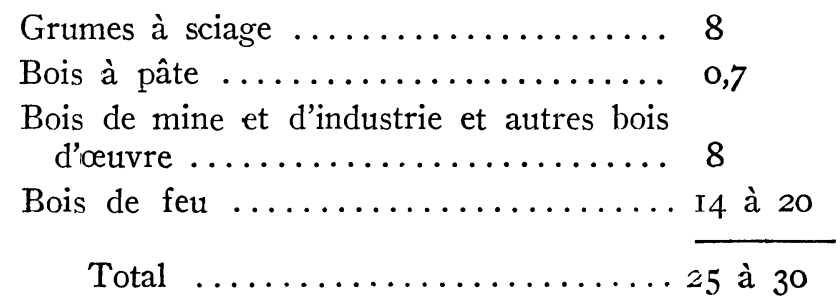

La forêt française, où dominent les propriétés privées, est trop riche en essences feuillues et produit trop de bois de feu.

Cette situation est bien connue des forestiers français qui, depuis plus d'un siècle, font porter leurs efforts sur les conversions de taillissous-futaie en futaies, sur l'enrésinement des taillis et sur la création de boisements résineux. Il n'y a pas lieu de citer ici les remarquables réussites obtenues à cet égard en France, réalisations que de nombreux forestiers étrangers viennent d'ailleurs visiter chaque année.

Le plan de reboisement pour la période 1950-1975, cité par la statistique de la F.A.O., prévoit pour la France la création de I 200000 hectares de forêts, soit I8 \% du programme européen. Le Fonds

(*) Consommation, Production et Commerce du Bois en Europe. Evolution et perspectives, Genève, 1953. Voir Rev. For. Franc., octobre 1953, p. 712 et sq. 
Forestier National a déjà permis d'amorcer avec succès ce vaste programme.

Mais il s'agit là d'une mesure à long terme, qui ne commencera à porter ses fruits que dans 20 ou 25 ans.

D'ores et déjà, en vue d'une augmentation immédiate de la production de bois à pâte, la F.A.O. recommande l'adoption d'une " politique forestière dynamique ), dont les grandes lignes sont:

- l'exploitation de forêts actuellement inaccessibles,

- l'extension des coupes d'éclaircie et autres améliorations sylvicoles, taires,

- le changement des possibilités à la suite de nouveaux inven-

- la récolte d'arbres hors forêt.

A cela s'ajoutent des mesures à caractère industriel telles que:

- la réduction des déchets dus à l'exploitation et au transport,

- l'utilisation industrielle des déchets des usines à bois.

Ces recommandations constituent les grandes lignes de la politique forestière européenne depuis de nombreuses années déjà : création de routes forestières, installation de câbles, révisions fréquentes des aménagements, calculs précis des possibilités, culture d'arbres hors forêt, surveillance attentive des exploitations ont constitué les préoçcupations essentielles de nombreuses générations de forestiers.

L'inventaire national des forêts françaises doit être refait (ce qui prouve que l'action des forestiers a été grande depuis l'établissement de la statistique Daubrée de I9I 2). Il permettra de connaitre avec précision la ressource en bois des diverses essences pour chacune des grandes catégories commerciales.

La majeure partie des bois de feu (14 millions de $\mathrm{m}^{3}$ ) est constituée par des essences feuillues. Mais ces bois de feucontiennent une forte proportion de produits non utilisables comme bois à pâtes: branches tordues, cimes noueuses, bois tarés. Si l'on admet que les 8 millions de $\mathrm{m}^{3}$ de grumes à sciages donnent en moyenne $0,5 \mathrm{~m}^{3}$ de bois de feu de houppier par $\mathrm{m}^{3}$ de fût, cela fait 4 millions de $\mathrm{m}^{3}$ de houppier, auxquels s'ajoutent les rémanents provenant du façonnage des 3,5 millions de $\mathrm{m}^{3}$ de bois d'industrie, rémanents qu'on peut estimer, très approximativement, à I million de $\mathrm{m}^{3}$. La production des bois de feu de taillis serait donc au minimum de 9 millions de $\mathrm{m}^{3}$. Ces taillis sont en majeure partie des essences suivantes: Chênes, Charme, Hêtre, Châtaignier, Aunes et Tremble.

Ils comportent des bois de diamètres variés: Chênes, Charme et Hêtre sont généralement de faible diamètre, le Tremble est souvent un peu plus gros, Châtaignier et Aune glutineux sont de diamètre nettement supérieur. 
Les Chênes et le Châtaignier contiennent une forte proportion de tanin. Ils donnent des pâtes brun-noirâtre qu'on ne peut guère utiliser que pour la couche intermédiaire des cartons. Les cossettes de Châtaignier détannées, que les usines d'extraits tannant utilisent pour faire des pâtes chimiques blanchies, proviennent presqu'uniquement des gros châtaigniers de vergers. Elles peuvent donner d'excellents papiers en mélange avec des pâtes résineuses.

Le Hêtre peut être parfaitement utilisé pour la fabrication de pâtes brunes, de pâtes mi-chimiques ou de pâtes au bisulfite. Il a d'ailleurs déjà fait l'objet d'essais et d'utilisations industriels. Un effort est à faire pour trouver une méthode d'écorçage économique.

Le Charme pourrait constituer une grosse ressource comme bois à pâtes, car il n'est pas très prisé comme bois de mine. Les dernières ventes dans l'Est ont permis de constater que les coupes riches en taillis de cette essence demeuraient invendus. Selon la Direction Générale des Eaux et Forêts, 50 \% des feuillus disponibles pour la papeterie en France sont des charmes. Le Charme possède de bonnes fibres (plus longues que celles des autres feuillus). Son bois est blanc. Il renferme 6r \% de fibres (à peu près la même chose que le Bouleau et le Tilleul) contre $57 \%$ pour le Chêne et le Châtaignier, et seulement $37 \%$ pour le Hêtre. Les parois ont une épaisseur $(3,7 \mu)$ inférieure à celle des parois des fibres du Chêne et du Hêtre (4,4 et $5,2 \mu)$, mais supérieure à celle du Châtaignnier et du Bouleau $(2,8 \mu)$ et surtout du Tremble ct du Tilleul $(2,4$ à $2, \mathrm{I} \mu)$.

La forme cannelée de la tige du Charme est défectueuse. Les rondins sont difficiles à écorcer. L'écorçage mécanique avec couteaux amène de grosses pertes de bois. Il serait particulièrement intéressant pour cette essence de mettre au point tune méthode d'écorçage chimique sur pied. Ia montée de sève chez cette espère est extrếmement précoce (février, dans l'Est de la France). On pourrait, à cette date, pratiquer l'annélation et l'application de poisons. Mais il y aurait lieu de choisir ceux-ci pour que, pendant les mois qui suivent cette opération, le bois, très sujet à l'échauffure, ne s'altère pas. Les rondins écorcés de Charme pourraient avoir, dans la fabrication des pâtes, les mêmes débouchés que ceux de Hêtre.

L'importance du Charme en France est grande (Io millions de stères par an). Aussi cette essence a-t-elle fait l'objet d'études très documentées à l'Ecole Française de Papeterie de Grenoble et aux laboratoires spécialisés de l'Association Technique de l'Industrie Papetière. Nous ne possédons pas les résultats complets de ces essais, mais l'examen des chiffres qui ont paru dans les rapports fait ressortir certaines qualités du Charme.

En ce qui concerne les pâtes obtentues par cuisson sodique (pâtes écrues ou pâtes raffinées), le Charme figure toujours en tête des feuillus, quant à la résistance à la déchirure, à la traction et à l'éclatement. Il se montre assez semblable aux Peupliers ou Tilleuls et 
légèrement supérieur au Bouleau. I1 est toujours nettement meilleur que les Chênes et le Juêtre.

I,e rapport $2 \mathrm{e} / \mathrm{Eh}$ du Charme est relativement fort (o,45 contre o,3 pour les Bouleau, Peupliers et Tilleuls): e étant l'épaisseur de la paroi en $\mu$ et Eh la largeur de la fibre humide, également en $\mu$.

Mais, la fibre sèche se torsade; ceci entraîne, pour le Charme une (" main ) un peu élevée (2,2 contre 1,9 aux Peupliers et Châtaignier; I,6 aux Tilleuls et I,5 à l'Epicéa). La main est le rapport entre l'épaisseur de la feuille en $\mathrm{mm}$ et le poids de la feuille en grammes par $\mathrm{m}^{2}$.

Malgré tout, cette essence reste susceptible d'une utilisation accrue, surtout dans le domaine des pâtes mi-chimiques.

Le Tremble est une essence intéressante, déjà parfaitement connue des papetiers, mais les trembles de nos forêts actuelles sont généralement de trop faible diamètre. Il s'agit d'une essence de lumière, craignant la concurrence des essences d'ombre. Ceux qu'on trouve dans les taillis d'essences mélangées sont généralement chétifs et dépérissants. La section de Génétique de la Station de Recherches Forestières de Nancy a déjà commencé la sélection de cette essence, opération qui entre dans le cadre des mesures à long terme pour l'amélioration de la production.

L'Aune glutineux est, lui aussi, un gros producteur de bois. Il a été essayé et même utilisé industriellement dans diverses fabriques de pâtes en France. Sa couleur rougeâtre constitue malheureusement un inconvénient, moins grave toutefois en cartonnerie qu'en papeterie.

Les taillis contiennent encore beaucoup d'autres espèces. Ce mélange constitue d'ailleurs un inconvénient pour l'emploi industriel en papeterie, car les propriétés des bois sont très variées et les fibres obtenues ne sont pas susceptibles des mêmes utilisations.

Cependant, certaines essences: Chênes, Châtaignier, Hêtre, Charme, existent en masses abondantes et peuvent constituer des approvisionnements susceptibles d'intéresser la papeterie. Les lessiveurs des usines de pâte chimique au bisulfite contiennent 200 à $2.50 \mathrm{~m}^{3}$ (le bois et, si l'on tient compte des vastes dimensions des appareils d'épuration et des machines à mettre les papiers et cartons en feuilles, on comprend que les approvisionnements en bois des usines de pâtes ne peuvent se faire que sous forme de volumes importants des mêmes essences.

Dans les taillis, on sera donc amené à trier les bois, ce qui augmentera le prix de façon. Pour vendre trembles, aunes, etc..., il faudra constituer des stères distincts dont le façonnage sera spécialement soigné : rondins bien calibrés, 'pas de bois tarés, pas de contacts avec le sol amenant terre, graviers et altérations. Afin de faciliter les manutentions ultérieures, il faudrait constituer des pa- 
quets (stères ou demi-stìres) cerclés au moyen de feuillards, comme les colis expédiés par fer. Ce perfectionnement devrait aller de pair avec celui des appareils mécaniques de chargement sur remorques, camions et wagons.

Ces sujétions relatives à la limitation de l'époque d'abattage, à l'écorçage, au tri des rondins et à leur empaquetage en colis cerclés, sont graves et elles auront de sérieuses répercussions sur le prix de revient. Compte tenu de l'infériorité des pâtes feuillues par rapport aux pâtes résineuses, et des difficultés de leur fabrication, il faut s'attendre à ce que le prix des bois de papeterie d'essences feuillues rendus usine, soit, en outre, sensiblement inférieur au prix des bois résineux. Aux U.S.A., les feuillus durs valent $25 \%$ de moins que les résineux. Tout ceci aura une répercussion sensible sur les prix sur pied des bois feuillus à fibres.

Mais si les producteurs font un effort pour l'amélioration du façonnage des bois de papeterie feuillus (études relatives à la mécanisation de l'abattage, à l'écorçage chimique, au transport, etc...) et s'efforcent de livrer à l'industrie une matière première de bonne qualité, ils voudraient être certains de trouver auprès de la papeterie des garanties de permanence et de régularité dans les achats, sans quoi rien ne peut être entrepris.

Grâce à cela, les forêts qui couvrent un cinquième de notre Pays verraient s'améliorer un peu l'avenir, actuellement sombre, pour certaines catégories de produits comme les petits bois d'essences dures.

J. VENET. 